\title{
Utilizando álgebras de flags para problemas de combinatória extremal
}

\author{
Roberto F. Parente ${ }^{1,2}$, Cristiane M. Sato ${ }^{3}$ \\ ${ }^{1}$ Departamento de Ciêcia da Computação - Universidade Federal da Bahia \\ Salvador, Brasil \\ ${ }^{2}$ Instituto de Matemática e Estatística - Universidade de São Paulo \\ São Paulo, Brazil \\ ${ }^{3}$ Centro de Matemática, Computação e Cognição - Universidade Federal do ABC \\ Santo André, Brazil \\ robertop@ime.usp.br, c.sato@ufabc.edu.br
}

\begin{abstract}
The goal of this paper is to present a simple introduction to the theory of flag algebras developed by Razborov, as well as illustrate this theory's power for obtaining results in extremal combinatorics. In this paper, we investigate a density problem in tournaments.
\end{abstract}

Resumo. O objetivo deste trabalho é apresentar uma introdução acessível à teoria das álgebras de flags desenvolvida por Razborov, bem como ilustrar a aplicabilidade desta teoria para obter resultados em combinatória extremal. Neste trabalho, lidamos com um problema de densidade em torneios.

\section{Introdução}

Neste trabalho, apresentamos uma breve introdução à teoria de álgebras de flag, desenvolvida por Razborov [Razborov 2007], que tem sido utilizada em diversos problemas de combinatória extremal. Para ilustrar a aplicabilidade dessa teoria, apresentamos um resultado para torneios [Coregliano et al. 2015, Parente 2016] obtido utilizando técnicas da teoria de álgebra de flags.

Um torneio é um digrafo $T=(V, A)$ tal que, para cada par não-ordenado de vértices $\{u, v\}$, temos que exatamente um dentre $(u, v)$ e $(v, u)$ pertence a $A$ (veja a Figura 1). O problema que estudamos é o seguinte. Seja $T$ um torneio com $t$ vértices. Queremos calcular o seguinte valor: $\max _{T_{n} \in \mathcal{T}_{n}} p\left(T, T_{n}\right)$, onde $\mathcal{T}_{n}$ é o conjunto de todos os torneios com $n$ vértices e $p\left(T, T_{n}\right)$ é o número de cópias de $T$ em $T_{n}$ dividido por $\left(\begin{array}{l}n \\ t\end{array}\right)$. Note que $p\left(T, T_{n}\right)$ é a probabilidade de, ao sortearmos um conjunto $S$ de $t$ vértices de $T_{n}$, obtermos um digrafo $T_{n}[S]$ isomorfo a $T$. Dizemos que $p\left(T, T_{n}\right)$ é a densidade de $T$ em $T_{n}$. Ou seja, queremos saber qual é a densidade máxima de $T$ considerando todos os torneios em $n$ vértices. Em combinatória extremal, é comum lidarmos com versões assintóticas dos problemas investigados. No nosso caso, isso significa que queremos calcular o valor $\lim _{n \rightarrow \infty} \max _{T_{n} \in \mathcal{T}_{n}} p\left(T, T_{n}\right)$. Neste trabalho, utilizamos álgebras de flag para mostrar que

$$
\lim _{n \rightarrow \infty} \max _{T_{n} \in \mathcal{T}_{n}} p\left(R, T_{n}\right)=1 / 16,
$$

The first author is supported by FAPESP grant (2016/24041-6) and this article was produced as part of the activities of FAPESP Research, Innovation and Dissemination Center for Neuromathematics (grant 2013/ 07699-0 , S.Paulo Research Foundation). The second author is Partially supported by FAPESP grant (Processo 2013/03447-6). 


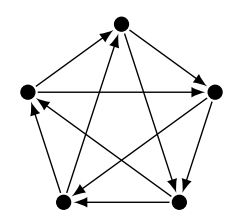

Figura 1. Torneio $R$ : um torneio em 5 vértices.

onde $R$ é o torneio na Figura 1. A prova completa do resultado está disponível em [Coregliano et al. 2015, Parente 2016].

Destacamos que uma característica de grande interesse no uso das álgebras de flags é a fácil utilização de resolvedores de programas semidefinidos para a obtenção de cotas superiores para os valores que queremos calcular.

\section{Álgebra de flags}

Nesta seção, apresentamos brevemente alguns conceitos de álgebra de flags. Para facilitar a leitura, definiremos flags no contexto de torneios. No entanto, um dos aspectos mais atrativos dessa teoria é que esta pode ser usada para modelar diversas estruturas combinatórias como, por exemplo, grafos, hipergrafos, permutações, grafos livres de uma família de grafos proibidos, entre outras. Mais precisamente, a teoria de álgebra de flags foi criada para lidar com qualquer teoria universal de primeira ordem.

A idéia principal é criar uma álgebra que capture as densidades $p\left(T, T_{n}\right)$. Trabalhamos com torneios rotulados e parcialmente rotulados: um tipo é um torneio cujo conjunto de vértices é $\{1, \ldots, t\}$ para algum $t \in \mathbb{N}$; dado um tipo $\sigma$ com $t$ vértices, uma $\sigma$-flag é um par $F=(T, \theta)$ onde $T$ é um torneio, $\theta:\{1, \ldots, t\} \rightarrow V(T)$ é uma imersão de $\sigma$ em $T$, ou seja, a função $\theta$ é um isomorfismo entre $\sigma$ e o torneio induzido por $\operatorname{im}(\theta)$ em $T$. Veja a Figura 2. Dado um tipo $\sigma$, denotamos por $\mathcal{F}_{\ell}^{\sigma}$ o conjunto das $\sigma$-flags com $\ell$ vértices e fazemos $\mathcal{F}^{\sigma}=\cup \mathcal{F}_{\ell}^{\sigma}$. Para $\sigma$-flags $T, T^{\prime}$ de tamanhos $t \leq t^{\prime}$, definimos $p\left(T, T^{\prime}\right)$ como a razão entre o número de conjuntos $W$ de tamanho $t-s$ (onde $s=|V(\sigma)|$ ) tais que $T^{\prime}[W \cup S]$ é isomorfo a $T$ (onde $S$ é o conjunto de vértices rotulados de $T$ ) e $\left(\begin{array}{c}t^{\prime} \\ t-s\end{array}\right)$.

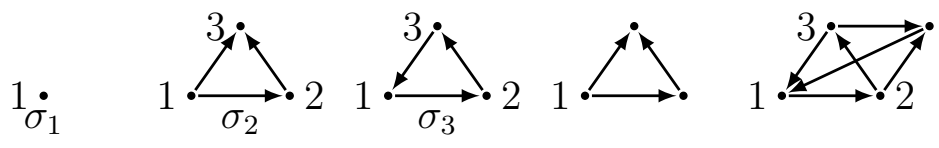

Figura 2. Tipos $\sigma_{1}, \sigma_{2}$ e $\sigma_{3}$, uma $\sigma_{1}$-flag e uma $\sigma_{3}$-flag

Em seguida, construímos o conjunto $\mathbb{R} \mathcal{F}^{\sigma}$ de todas as combinações lineares formais dos elementos de $\mathcal{F}^{\sigma}$. Por exemplo, se $T$ e $T^{\prime}$ são $\sigma$-flags, então $T+T^{\prime}, T-T^{\prime}, 4 T-$ $(2 / 3) T^{\prime}$ são elementos de $\mathbb{R} \mathcal{F}^{\sigma}$ (ou seja, qualquer combinação com quaisquer coeficientes é válida). No entanto, esse espaço de combinações não captura as densidades como queríamos e não mostra nenhuma relação entre as combinações. Para capturar as densidades, gostaríamos que $T$ fosse considerado igual à combinação linear $\sum_{T_{n} \in \mathcal{F}_{n}^{\sigma}} p\left(T ; T_{n}\right) T_{n}$, pois $p\left(T, T_{n}\right)=\sum_{\tilde{T} \in \mathcal{F}_{n}^{\sigma}} p(T ; \tilde{T}) p\left(\tilde{T}, T_{n}\right)$. Isso pode ser feito da seguinte maneira. Considere o subsepaço linear $\mathcal{K}^{\sigma}$ gerado pelos elementos $T-\sum_{T_{n} \in \mathcal{F}_{n}^{\sigma}} p\left(T ; T_{n}\right) T_{n}$ (para todo $n \in \mathbb{N}$ com $n \geq V(T)$ ). Queremos considerar todos os elementos de $\mathcal{K}^{\sigma}$ como zero. 
Isso é feito definindo $\mathcal{A}^{\sigma}=\mathbb{R} \mathcal{F}^{\sigma} / \mathcal{K}^{\sigma}$ como a álgebra ${ }^{1}$ obtida pelo quociente de $\mathbb{R} \mathcal{F}^{\sigma}$ por $\mathcal{K}^{\sigma}$. Por exemplo, seja $T$ uma $\sigma$-flag com $t$ vértices e $T^{\prime}$ uma $\sigma$-flag com $t^{\prime}$ vértices. Como poderíamos avaliar a expressão $T-T^{\prime}$ ? Seja $n \geq \max \left\{t, t^{\prime}\right\}$ e suponha que $\sum_{T_{n} \in \mathcal{F}_{n}^{\sigma}} p\left(T ; T_{n}\right)=(1 / 2) T_{a}+(1 / 2) T_{b}$ e $\sum_{T_{n} \in \mathcal{F}_{n}^{\sigma}} p\left(T^{\prime} ; T_{n}\right)=(1 / 3) T_{a}+(2 / 3) T_{b}$. Então na álgebra vale que $T-T^{\prime}=(1 / 6) T_{a}-(1 / 6) T_{b}$.

Agora volte a considerar o problema de mostrar que $\lim _{n \rightarrow \infty} \max _{T_{n} \in \mathcal{T}_{n}} p\left(R, T_{n}\right)$ é $1 / 16$, onde $R$ é o torneio na Figura 1. Uma propriedade interessante é que existe uma sequência $\left(T_{n}\right)_{n \in \mathbb{N}}$ de torneios com tamanho crescente tal que não apenas $\lim _{n \in \mathbb{N}} p\left(T, T_{n}\right)=1 / 16$, mas $\lim _{n \in \mathbb{N}} p\left(T^{\prime}, T_{n}\right)$ também existe para todo torneio $T^{\prime}$. Assim, é natural dizer que uma sequência $\left(T_{n}\right)_{n \in \mathbb{N}}$ de $\sigma$-flags é convergente se é crescente e para toda $\sigma$-flag fixa $F \in \mathcal{F}^{\sigma}$, a sequência de números reais $\left(p\left(F ; F_{n}\right)\right)_{n \in \mathbb{N}}$ é convergente. Ou seja, o nosso problema é equivalente a calcular $\lim _{n \rightarrow \infty} p\left(T, T_{n}\right)$ onde $\left(T_{n}\right)_{n \in \mathbb{N}}$ é uma sequência convergente.

Uma parte essencial da teoria de álgebra de flags é lidar com essas sequências convergentes. O que seria um objeto-limite apropriado? Se $\left(T_{n}\right)_{n \in \mathbb{N}}$ é uma sequência convergente de $\sigma$-flags, precisamos de um objeto $\phi$ para o qual e $\phi(T)=\lim _{n \rightarrow \infty} p\left(T ; T_{n}\right)$. Seja $\operatorname{Hom}^{+}\left(\mathcal{A}^{\sigma}, \mathbb{R}\right)$ homomorfismos de álgebras ${ }^{2} \phi$ de $\mathcal{A}^{\sigma}$ para $\mathbb{R}$ tal que $\phi(T) \geq 0$ para toda $\sigma$ flag $T$. Dizemos que uma sequência de $\sigma$-flags $\left(T_{n}\right)_{n \in \mathbb{N}}$ converge para $\phi \in \operatorname{Hom}^{+}\left(\mathcal{A}^{\sigma}, \mathbb{R}\right)$ se $\phi(T)=\lim _{n \rightarrow \infty} p\left(T ; T_{n}\right)$ para toda $\sigma$-flag $T$.

Como saber que esses homomorfismos são o objeto-limite "certo" para as sequencias convergentes? Um belo resultado de Razborov [Razborov 2007, Theorem 3.3] e de Lovász e Szegedy [Lovász and Szegedy 2006] nos diz que toda sequência convergente de $\sigma$-flags converge para um homomorfismo em $\operatorname{Hom}^{+}\left(\mathcal{A}^{\sigma}, \mathbb{R}\right)$. Por outro lado, para todo homomorfismo $\phi \in \operatorname{Hom}^{+}\left(\mathcal{A}^{\sigma}, \mathbb{R}\right)$, existe uma sequência de $\sigma$-flags que converge para $\phi$. Assim, temos que

$$
\lim _{n \rightarrow \infty} \max _{T_{n} \in \mathcal{T}_{n}} p\left(R, T_{n}\right)=\max _{\phi \in \operatorname{Hom}^{+}\left(\mathcal{A}^{0}, \mathbb{R}\right)} \phi(T), \quad \text { onde } \mathbf{0} \text { é um torneio sem vértices. }
$$

\section{O método semidefinido para álgebra de flags}

Note que prover limitantes inferiores para problemas de densidade máxima é uma tarefa relativamente fácil. De fato, toda sequência crescente de torneios $\left(T_{n}\right)_{n \in \mathbb{N}}$ nos dá um limitante inferior $\lim \sup _{n \rightarrow \infty} p\left(T ; T_{n}\right)$. De fato, seja $R_{2 n+1}$ o que chamaremos de torneio carrossel. Esse torneio tem conjunto de vértices $V\left(R_{2 n+1}\right)=\{0,1, \ldots, 2 n\}$ e conjunto de $\operatorname{arcos} A\left(R_{2 n+1}\right)=\left\{(v,(v+i) \bmod (2 n+1)): v \in V\left(R_{2 n+1}\right)\right.$ e $\left.i \in[n]\right\}$, onde $[n]=\{1,2, \ldots, n\}$. Através de uma prova combinatória simples, pode-se mostrar que $\lim _{n \rightarrow \infty} p\left(R, R_{n}\right)=1 / 16$. Mas como mostrar que não é possível atingir um valor maior? De fato, essa é a parte difícil da prova.

Na seção anterior, vimos que o problema de calcular a densidade máxima de $R$ pode ser visto como um problema de maximização em $\operatorname{Hom}^{+}\left(\mathcal{A}^{\sigma}, \mathbb{R}\right)$. À primeira vista, pode parecer que trocamos um problema relativamente simples de descrever e de forte

\footnotetext{
${ }^{1}$ Para que $\mathcal{A}^{\sigma}$ seja de fato uma álgebra, é preciso que definir também um produto. Para detalhes, veja o artigo [Coregliano et al. 2015, Parente 2016].

${ }^{2}$ Uma função $\phi: \mathcal{A}^{\sigma} \rightarrow \mathbb{R}$ é um homomorfismo de álgebra se para todo $\alpha \in \mathbb{R}$ e $f, g \in \mathcal{A}^{\sigma}$, vale que $\phi(\alpha \cdot f)=\alpha \cdot \phi(f), \phi(f \cdot g)=\phi(f) \cdot \phi(g)$ e $\phi(f+g)=\phi(f)+\phi(g)$.
} 
intuição combinatória por um problema muito mais difícil por lidar com objetos muito mais complexos (os homomorfismos). De fato, a intuição combinatória perde-se nos homomorfismos. No entanto, Razborov desenvolveu um método para formular problemas semidefinidos para obter limitantes superiores para (2). Dessa forma, podemos usar o auxílio do computador por meio de resolvedores para programas semidefinidos para obter esses limitantes. Esse método tem sido usado com muito sucesso para avançar o entendimento de diversos problemas de combinatória extremal.

A seguir, descrevemos como construir o programa semidefinido para o nosso problema. Primeiro, escolhemos um número $m \in \mathbb{N}$ e tipos $\sigma_{1}, \ldots, \sigma_{t}$. Para cada $1 \leq i \leq m$, seja $k_{i}$ o número de vértices de $\sigma_{i}$. Em seguida, escolhemos números $\ell, \ell_{1}, \ldots, \ell_{m}$ tais que $2 \ell_{i}-k_{i} \leq \ell$ para todo $1 \leq i \leq m$.

No programa semidefinido abaixo, as variáveis são matrizes $Q(i) \in \mathbb{R}^{\mathcal{F}_{\ell_{i}}^{\sigma_{i}} \times \mathcal{F}_{\ell_{i}}^{\sigma_{i}}}$, para $1 \leq i \leq m$. Uma matriz simétrica real é dita positiva semidefinida se todos os seus autovalores são não-negativos. Para duas matrizes $A, B \in \mathbb{R}^{n \times n}$, o produto interno $\langle A, B\rangle$ é definido como $\sum_{i=1}^{n} \sum_{j=1}^{n} A_{i, j} B_{i, j}$. O programa semidefinido é o seguinte:

$\min y$

$$
\begin{array}{ll}
\text { s.t. } \quad p(R, T)+\sum_{i=1}^{m}\langle Q(i), C(i, T)\rangle \leq y \quad \forall T \in \mathcal{T}_{\ell} ; \\
Q(i) \in \mathbb{R}^{\mathcal{F}_{\ell_{i}}^{\sigma_{i}} \times \mathcal{F}_{\ell_{i}}^{\sigma_{i}} \text { é positiva semidefinida }} \quad \forall i \in\{1, \ldots, m\},
\end{array}
$$

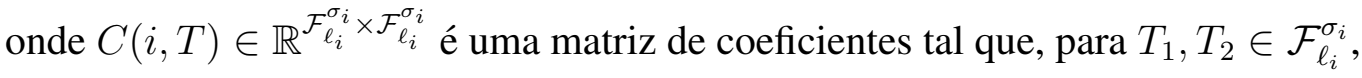

$$
C(i, T)_{T_{1}, T_{2}}=\sum_{T \in \mathcal{F}_{\ell}^{\sigma_{i}}} p\left(T_{1}, T_{2} ; T\right) p\left(\sigma_{i},\left.T\right|_{0}\right) p\left(\left.T\right|_{0}, T\right),
$$

onde $\left.T\right|_{0}$ é o torneio obtido a partir de $T$ após a remoção dos rótulos e $p\left(T_{1}, T_{2} ; T\right)$ é a densidade conjunta de $T_{1}$ e $T_{2}$ em $T$, que é definida por $p\left(T_{1}, T_{2} ; T\right)=$

$|W| /\left(\frac{\ell !}{\left(\ell_{i}-k_{i}\right) !^{2}\left(\ell-2 \ell_{i}-2 k_{i}\right) !}\right)$, onde $W$ é o conjunto de pares $\left(W_{1}, W_{2}\right)$ de subconjuntos disjuntos de $V(T)$ de tamanho $\ell_{i}-k_{i}$ tais que $T\left[W_{1} \cup S\right] \cong T_{1}$ e $T\left[W_{2} \cup S\right] \cong T_{2}$ e $S$ é o conjunto de vértices rotulados de $T$. Claramente, computar esses coeficientes é uma parte trabalhosa para a aplicação do método. Para o nosso problema, escolhemos $m=3$, $\ell=5, \ell_{1}=3, \ell_{2}=\ell_{3}=4$. Os tipos $\sigma_{1}, \sigma_{2}$ e $\sigma_{3}$ estão na Figura 2. Com isso, obtemos um programa semidefinido e utilizamos os programas CDSP e SDPA para obter o valor 1/16.

\section{Referências}

[Coregliano et al. 2015] Coregliano, L. N., Parente, R. F., and Sato, C. M. (2015). On the maximum density of fixed strongly connected subtournaments. ArXiv e-prints.

[Lovász and Szegedy 2006] Lovász, L. and Szegedy, B. (2006). Limits of dense graph sequences. J. Combin. Theory Ser. B, 96(6):933-957.

[Parente 2016] Parente, R. F. (2016). Empacotamento e contagem em digrafos: cenários aleatórios e extremais. PhD thesis, Instituto de Matemática e Estatística, Universidade de São Paulo.

[Razborov 2007] Razborov, A. (2007). Flag algebras. J. Symbolic Logic, 72(4):1239-1282. 